\title{
Modeling of GalnP/GaAs Dual-Junction Solar Cells including Tunnel Junction
}

\author{
Mathieu Baudrit and Carlos Algora \\ Instituto de Energía Solar, Universidad Politécnica de Madrid, Spain \\ mbaudrit@ies-def.upm.es
}

\begin{abstract}
This paper presents research efforts conducted at the IES-UPM in the development of an accurate, physically-based solar cell model using the generalpurpose ATLAS $^{\circledR}$ device simulator by Silvaco. Unlike solar cell models based on a combination of discrete electrical components, this novel model extracts the electrical characteristics of a solar cell based on virtual fabrication of its physical structure, allowing for direct manipulation of materials, dimensions, and dopings. As single junction solar cells simulation was yet achieved, the next step towards advanced simulations of multi-junction cells (MJC) is the simulation of the tunnel diodes, which interconnect the subcells in a monolithic MJC. The first results simulating a DualJunction (DJ) GalnP/GaAs solar cells are shown in this paper including a complete Tunnel Junction (TJ) model and the resonant cavity effect occurring in the bottom cell. Simulation and experimental results were compared in order to test the accuracy of the models employed.
\end{abstract}

Index Terms: Multi-Junction Solar Cells, Tunnel Junction, Simulation

\section{INTRODUCTION}

LEDs, lasers and Multi-junction Solar Cells can all employ tunnel junctions to improve performance. Calculating the effects of this junction is tricky, but there are ways to accurately simulate chip characteristics and cost-effectively optimize the structure's design. After the successful simulation of III/V single junction solar cells [1], we tested the known tunneling models such as Hurkx, Kane and Klaassen. Despite their good behavior in reverse bias they do not manage to reproduce the JV curve of a tunnel diode in forward bias.

An intense work was then done to create a reliable and flexilble model to allow the complete electrical simulation of tunnel diodes, especially in forward bias. After the development of a non-local tunneling model, room was opened to Dual-Junction solar cell modeling. In this paper we present the first results obtained by IES-UPM regarding MJC simulation using Silvaco ATLAS $^{\circledR}$. We show that when correctly adjusted, the software allows a good fit between simulation and experimental results for both Tunnel Junction and Dual-Junction solar cell.

\section{TUNNEL JUNCTION MODEL}

The local band-to-band tunneling models use the electric field value at each node to give a generation rate at that point due to the tunneling. In reality, the tunneling process is non-local and is necessary to take into account the spatial profile of the energy bands. It is also necessary to take into account the spatial separation of the electrons generated in the conduction band from the holes generated in the valence band [2].

A model for this process has been created for ATLAS ${ }^{\circledR}$ [3]. It assumes that the tunneling can be modeled as being one-dimensional in nature so that it can be calculated using a special rectangular mesh superimposed over and coupled to the ATLAS ${ }^{\circledR}$ mesh. This mesh needs to include the junction region of interest and the direction of the band-to-band tunneling, which is generally perpendicular to junction interface, must be specified.

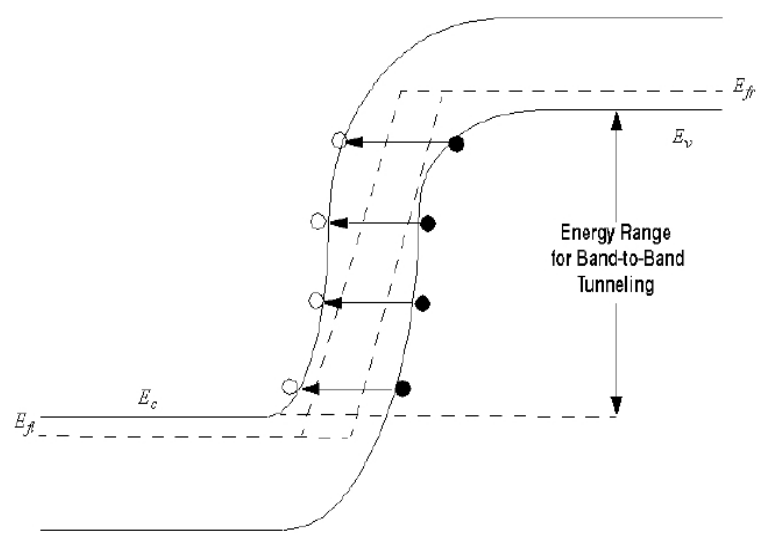

Figure 1. Schematic band diagram of a tunnel junction.

In order to explain how the tunneling current is calculated, let us consider an energy band profile along each slice in the tunneling direction when applying a reverse bias across the junction. Figure 2 shows a schematic of this, together with the allowed range of valence band electron energy for which tunneling is permitted.

At moderate doping levels, a tunneling effect can be seen in reverse bias, but if the junction doping levels are high enough, then this energy range may also exist in forward bias and tunneling effect can also be appreciated. If we consider only elastic scattering mechanisms, then electrons from anywhere in the permitted energy range can tunnel from the valence band to the conduction band. ATLAS ${ }^{\circledR}$ considers each 
energy in the allowed range and determines the spatial start and end positions for the tunneling at each energy, $E$, which we label $x_{b e g}$ and $x_{e n d}$. respectively, the contribution to tunneling current for an electron in the energy range from $E-\Delta E / 2$ to $E+\Delta E / 2$ (where $\Delta E$ is a small energy increment) is

$$
J(E)=\frac{q K T}{2 \pi^{2} h^{3}} T(E)\left\{m_{e}{ }^{*} \ln \left\{\frac{1+\exp \left[\left(E-E_{F l}^{e}\right) / k T\right]}{1+\exp \left[\left(E-E_{F r}^{e}\right) / k T\right]}\right\}-m_{h}{ }^{*} \ln \left\{\frac{1+\exp \left[\left(E-E_{F l}^{h}\right) / k T\right]}{1+\exp \left[\left(E-E_{F r}^{h}\right) / k T\right]}\right\} \Delta E\right.
$$

(1)

where $T(E)$ is the tunneling probability, $T$ the temperature, $E_{f l}$ and $E_{f r}$ are defined on Figure 1 and

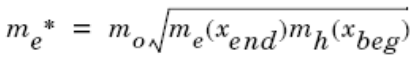

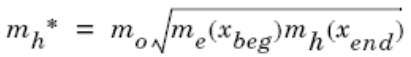

In equilibrium, $E_{f l}=E_{f r}$ and the current is zero as expected. This contribution to the tunneling current is calculated and coupled into the mesh at $x_{\text {beg }}$ and $x_{\text {end }}$. ATLAS $^{\circledR}$ uses a transmission matrix method to calculate the tunneling probability for direct quantum tunneling simulations through an insulator. In the case of band-to-band tunneling, however, a carefully applied Wenzel-Kramers-Brillouin (WKB) method was found to give equivalent results and is computationally more efficient.

\section{TJ: COMPARISON WITH EXPERIMENTAL RESULTS}

In order to calibrate the nonlocal tunnel model, an isolated $\mathrm{p}^{++}$-GaAs / $\mathrm{n}^{++}$-GaAs tunnel diode was grown by MOVPE has been investigated. The simulated structure is shown in Figure 2.

The tunnel diode consists of the tunnel junction itself made by two degenerately doped $n^{++}$- and $p^{++}-\mathrm{GaAs}$ layers as well as two enclosing barrier layers with the purpose to minimize dopant diffusion [4]. The cap layer and the substrate have been included in the simulation. For the purpose of this paper, a tunnel diode with a sharp uniform doping profile was modeled.

Local and nonlocal Trap Assisted Tunneling (TAT) mechanisms have been included in the models involved in tunneling effects because of their influence on the simulated IV curve.

Figure 3 resumes how the TAT works in the case of tunnel junction. It is indeed divided in two different effects: the local one affecting mainly the peak current and the decreasing slope of the IV curve and the non- local one, affecting the current after the $J_{\text {valley }}$ and increasing its value

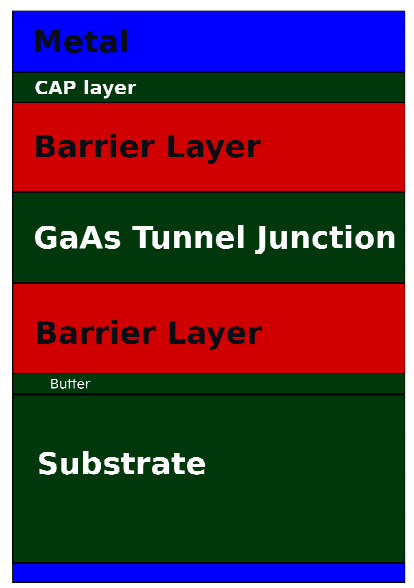

Figure 2: Simulated tunnel junction

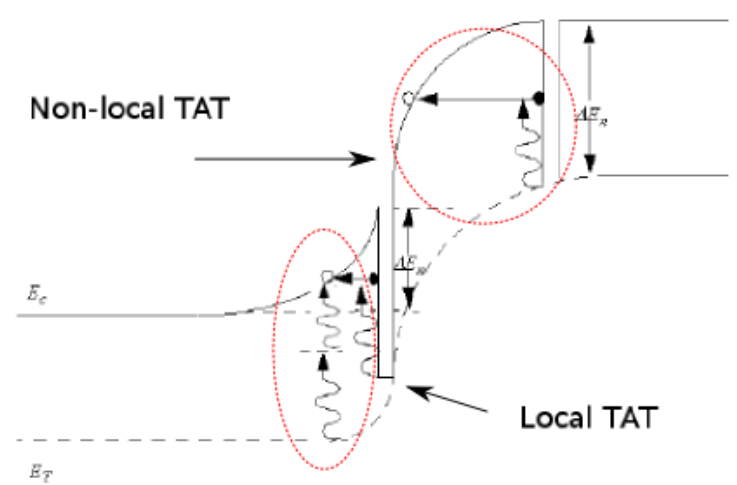

Figure 3: Trap Assisted Tunneling mechanisms.

Figure 4 shows the very good fit obtained as a result of the tunnel junction simulation made with Silvaco ATLAS $^{\circledR}$ software including the nonlocal tunneling model described before as well as TAT and nonlocal TAT. Results have been obtained after adjusting the material parameters affecting the tunneling effect such as the effective mass for both holes and electrons, the trap concentration and the limits of the superimposed mesh. In this case, a high specific contact resistance was put in evidence, reaching actually the value of $0.3 \mathrm{e}-3 \mathrm{mohm} \cdot \mathrm{cm}^{2}$ in the case of the tunnel junction presented in figure 4 but reaching sometimes the value of $1.5 \mathrm{e}-2 \mathrm{ohm} \cdot \mathrm{cm}^{2}$. This very high value is due to the metal/semiconductor contact, actually we saw that the metallization grid on some measured tunnel diode was of very poor quality, inducing then a very high series resistance. We also have to note that the real doping profile, which has been measured for other tunnel junctions, is important to allow a faster fit between experimental and simulation data. 


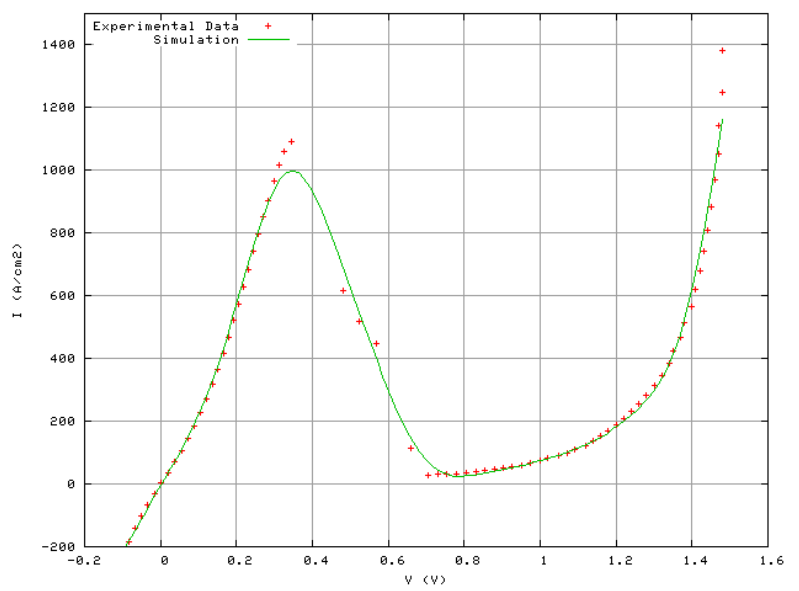

Figure 2: Experimental (dots) and simulated (solid line) Tunnel Junction IV curve.

\section{DUAL-JUNCTION SOLAR CELL SIMULATION}

Once we are able to simulate the tunnel diode, the next step is the modeling of a complete Dual-Junction solar cell. We will focus in this paper on the External Quantum Efficiency (EQE), the IV curve at 1 sun and the dark IV curve.

\section{External Quantum Efficiency}

In a GalnP/GaAs dual-junction solar cell, GaAs bottom cell suffers oscillations of its External Quantum Efficiency because of the resonant cavity effect occurring between the top cell BSF and the bottom cell window layer. In this case, using traditional ray-tracing is useless because reflected rays in each layer should be set to a very large number and simulation time increases exponentially with the internal reflection number. The only way to achieve a good accuracy is to use the Characteristic Matrix Method or the Transfer Matrix Method.

Silvaco ATLAS $^{\circledR}$ uses the Characteristic Matrix approach that relates total tangential components of the electric and magnetic fields at the multilayer boundaries. The structure of a multilayer completely determines the characteristic matrix of this multilayer. The transfer matrix also contains information about the media on both sides of the multilayer.

\begin{tabular}{|c|c|c|c|}
\hline Name & Material & Thickness & $\mathrm{N}\left[\mathrm{cm}^{-3}\right]$ \\
\hline topfsf & AllnP & $50 \mathrm{~nm}$ & $\mathrm{n}=3.010^{+17}$ \\
\hline topem & $\mathrm{Ga}_{0.51} \ln _{0.49} \mathrm{P}$ & $170 \mathrm{~nm}$ & $\mathrm{n}=1.810^{+18}$ \\
\hline topbase & $\mathrm{Ga}_{0.51} \mathrm{In}_{0.49} \mathrm{P}$ & $800 \mathrm{~nm}$ & $p=1.010^{+17}$ \\
\hline topbsf & AlGalnP & $100 \mathrm{~nm}$ & $p=3.010^{+17}$ \\
\hline phighTD & GaAs & $50 \mathrm{~nm}$ & $p=5.010^{+19}$ \\
\hline nhighTD & GaAs & $50 \mathrm{~nm}$ & $\mathrm{n}=3.010^{+19}$ \\
\hline botfsf & $\mathrm{Al}_{0.4} \mathrm{Ga}_{0.6} \mathrm{As}$ & $50 \mathrm{~nm}$ & $\mathrm{n}=2.010^{+18}$ \\
\hline botem & GaAs & $100 \mathrm{~nm}$ & $\mathrm{n}=1.010^{+10}$ \\
\hline botbase & GaAs & $3500 \mathrm{~nm}$ & $p=2.010^{+17}$ \\
\hline botbsf & $\mathrm{Al}_{0.3} \mathrm{Ga}_{0.7} \mathrm{As}$ & $100 \mathrm{~nm}$ & $p=2.010^{+18}$ \\
\hline subs & GaAs & $300 \mu \mathrm{m}$ & $p=2.010^{+18}$ \\
\hline
\end{tabular}

Table 1. Nominal semiconductor structure of the Dual-Junction solar cell.

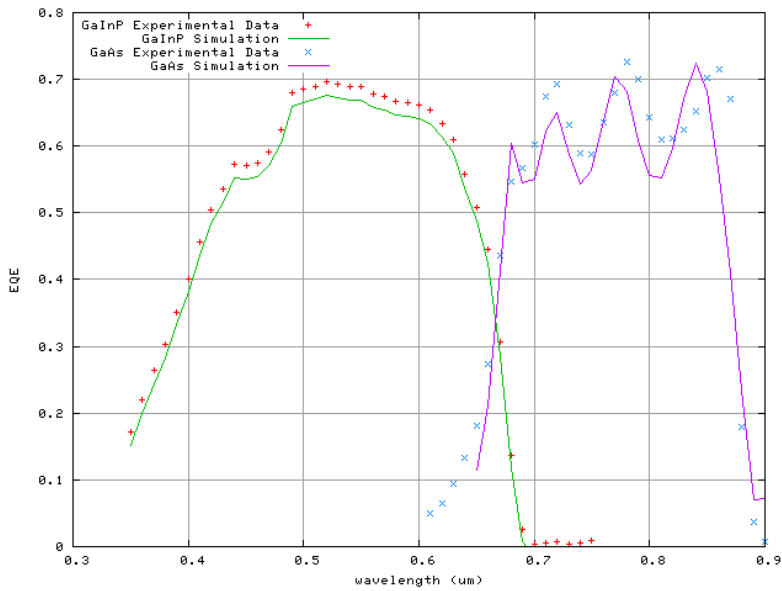

Figure 3: Experimental (dots) and simulated (solid lines) External Quantum Efficiency of the Dual-Junction solar cell.

Table 1 describes the layer structure of the simulated Dual-Junction solar cell. The results in Figure 5 show a good agreement with experimental data. The small mismatch is probably due to the differences between nominal thicknesses (introduced in the simulation) and real values which were not measured exactly. We will further make an in depth characterization (Doping concentration and thicknesses) to perfect the fit between experimental and simulated results.

\section{JV curve at 1 sun}

Once the EQE simulation agreed well with the measurement and we can do do a good $J_{s c}$ estimation using a simulation software, it was necessary to achieve an JV curve simulation to see how the models can predict the $V_{o c}$, the fill factor and the efficiency of the solar cell.

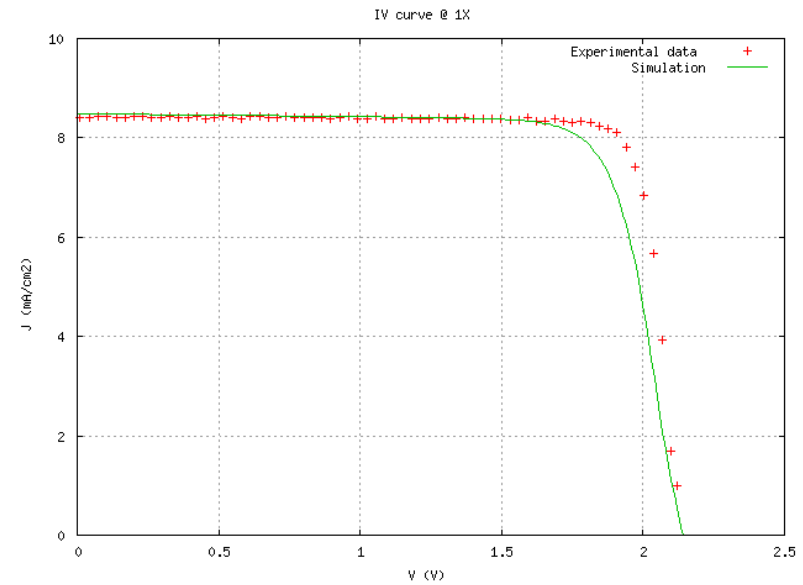

Figure 4: Experimental (dots) and simulated (solid line) IV curve

As demonstrated before by the EQE simulation, we see a very good fit of the $J_{s c}$ using the AM1.5 LowAOD spectra (see Figure 6), however, slight 
disagreements can be observed. Table 2 below resumes the main characteristics of the cell.

\begin{tabular}{|l|c|c|}
\hline & $\begin{array}{c}\text { Experimental } \\
\text { results }\end{array}$ & Simulation \\
\hline $\mathrm{Jsc}\left(\mathrm{mA} / \mathrm{cm}^{2}\right)$ & 8.4 & 8.4 \\
$\operatorname{Voc}(\mathrm{V})$ & 2.12 & 2.12 \\
$\mathrm{Jmax}\left(\mathrm{mA} / \mathrm{cm}^{2}\right)$ & 7.9 & 7.66 \\
$\operatorname{Vmax}(\mathrm{V})$ & 1.9 & 1.82 \\
$\mathrm{FF}(\%)$ & 84 & 78 \\
$\eta(\%)$ & 15 & 13.9 \\
\hline
\end{tabular}

Table 2: Parameters extracted from the experimental and simulated IV curve

The DJ solar cell simulated had no Anti-Reflective Coating layers and is a low/medium quality solar cell which can explain the low efficiency at 1 Sun and shows that the models are not only able to reproduce high quality solar cells but also lower quality devices.

Once again the simulation shows a very good fit with the experimental data on the $V_{o c}$ prediction. However, some differences can be seen on the FF value and so on on the $J_{\max }, V_{\max }$ and Efficiency.

As good results were obtained before modeling single junction solar cells at IES-UPM, we suspected the tunneling model used in the DJ solar cell. To be exact we suspected that the model doesn't behave as it should under illumination, this why we simulated a dark IV curve to localize the origin of the differences under illumination.

\section{Dark JV curve}

In contrast with the other DJ solar cells simulations presented in this paper, the dark IV curve only relies on the electrical models as no light is input to the device.

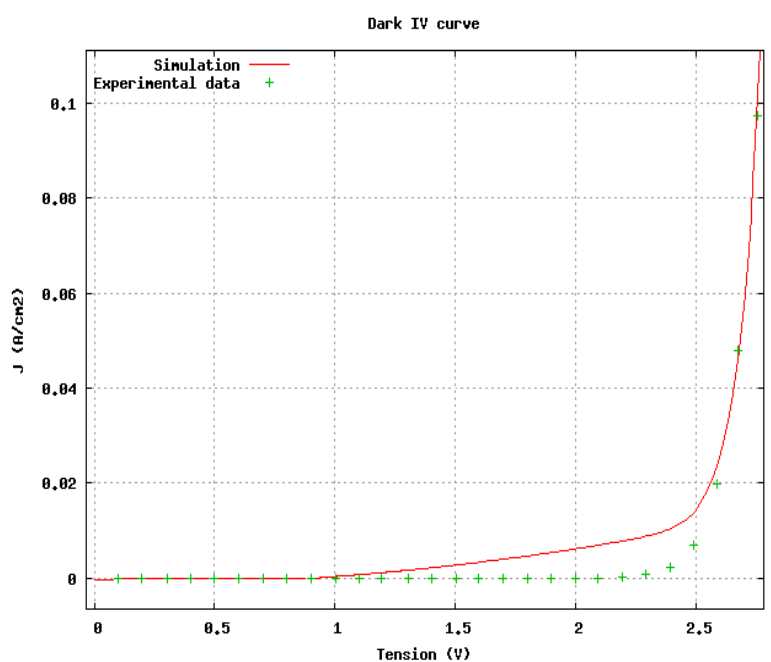

Figure 5: Experimental (dots) and simulated (solid line) dark IV curve
As seen before with the IV curve under illumination, there is a misfit between experimental and simulation data, especially for middle range voltage. If we compare Figure 6 and Figure 7, we also see the mismatch voltage range is different but the dark JV also shows a shunt resistance effect.

As in this case there is no light input to the device and differences can be observed, light interaction as nothing to do with this. However, viewing the results and the good results obtained before simulating single junction solar cells, we think the mismatch is due the tunnel model once introduced inside a complete dualjunction solar cell structure. More, we think this is due to how the tunneling model manage traps as we saw the kind of curve seen in Figure 7 in some structures with a very high traps concentration, with the difference that in our case the traps concentration cannot explain such a difference.

The revision of the tunnel model will be our priority in the next months as, despite of the good results, we need it to be totally reliable.

\section{CONCLUSIONS}

Before the existence of reliable non-local tunneling model, the simulations of dual-junction solar cells were limited to optically coupled simulations, separating then the cells electrically. With the development of a reliable non-local tunneling model, a complete electrical simulation is now possible. It was shown that in contrast to local tunneling models the nonlocal tunneling model reproduces the measured JV curve of a tunnel diode structure in a large voltage range very well, especially in the decisive range of operation when applied to multi-junction solar cells.

We also proved the validity of the model, which coupled with an adequate optical modeling method, allows to reproduce very well the EQE of a DJ solar cell, making the $J_{s c}$ prediction accurate. Regarding the JV curves, despite the good results obtained on $V_{o c}$ estimation, we detect a problem affecting the values of the FF and the efficiency. We think this problem is due to the tunneling model behavior and a revision of it has to be done.

However, this paper shows that solar cells simulation under a TCAD environment is possible and can be predictive for single junction solar cells as well as for DJ solar cells. Room is open for Multi-Junction solar Cells simulation as we now know the critical part of this structure, the tunnel junction, is well managed.

The future works at the IES-UPM will be the enhancement of the simulated structure to triple junction solar cells and the inclusion of real operation conditions as they can strongly change the optimization of the cell.

\section{Acknowledgements}

The authors would like to thank the Silvaco Grenoble Research Center (G.RE.CE.) for its collaboration. Thanks also to Ignacio Rey-Stolle, Beatriz Galiana, Iván García and Pilar Espinet of the IES-UPM who made the experimental data possible. 
This paper has been supported by the European Commission under contract SES6-CT-2003-502620 (FULLSPECTRUM project). The Spanish Ministerio de Educación y Ciencia has also contributed with the CONSOLIDER- INGENIO 2010 program by means of the GENESIS FV project (CSD2006-004) and also with the research projects with references TEC2005-02745 and TEC2004-22300-E as well as the Comunidad de Madrid under NUMANCIA programme (S05050/ENE/0310).

\section{REFERENCES}

[1] C.Algora, et al, Pending Issues In The Modeling of Concentrator Solar Cells, Proceedings 19th European Photovoltaic Solar Energy Conference, Paris, 2004.

[2] G. A. M. Hurkx, D. B. M. Klaassen, and M. P. G. Knuvers, A new recombination model for device simulation including tunneling in IEEE Transactions on Electron Devices 39, 331-8 (1992).

[3] Silvaco International, Silvaco User's Manual, ed. Silvaco, 2006.

[4] T. Takamoto, et al, Mechanism of $\mathrm{Zn}$ and $\mathrm{Si}$ diffusion from a highly doped tunnel junction for InGaP/GaAs tandem solar cells in Journal of Applied Physics 85, 1481-6 (1999). 